\title{
The Structural Pattern and Promotion Mechanism of Circular Economy Based on Topological Theory
}

\author{
Kui Yu \\ School of Economics and Management, Yibin University, Yibin, 644000, China
}

Keywords: Circular Economy, Topological Structure, Operation Mechanism, Ecological Industry

\begin{abstract}
Circular economy is the concept of economic development which gains general consent, but as a new economic model which differs from the traditional linear development, the practices in many places have the problems of instability and unsustainability in our country. This text explores the structure of the circular economic system in our country, analyzes its components, types and formation mode and provides the stable structure which can maintain the recycling system and the promotion mechanism which can realize the sustainable development by using the topological knowledge in mathematics.
\end{abstract}

\section{The Meaning of Topological Structure of the Circular Economic System}

The topological structure of circular economic system abstracts the enterprises, infrastructure and public service inside the system into a point by adopting the method of research and size, the points irrelevant to the shape and the linear relationship in topology, then it abstracts the connection of various entities into a line. The geometric figure formed by points and lines is the topological structure of circular system. ${ }^{[1]}$ The topological structure of circular economic system, which is the first step to construct the circular economy system, reflects the structural relationship of various entities in the circular economy system and it has significant impact on the stability, balance, sustainability and economic interests of the circular economic system.

\section{Node}

The node of the topological structure of circular economy system, which is formed by various enterprises, is the basic production and switching unit of matter energy and also the basic material condition to form the circular economy.

According to the different industries, the node can be divided into homogeneous node and heterogeneous mode.

Homogeneous node means the enterprises belonging to the same industries and they usually have relatively same material input, production output and production technology. The circular economy systems whose topological structure is formed by homogeneous node mostly are the industry-type industrial park of circular economy such as the demonstration park of the national eco-industrial construction in Guigang, Guangxi leaded by sugar industry.[2]

Heterogeneous node means the enterprises belonging to different industries. The circular economy systems formed by heterogeneous nodes mostly are the regional industrial parks of circular economy. These industrial parks are the new planning or transformation. The classical representative is the demonstration park of the national eco-industrial construction in Huangxing, Changsha.

According to the interaction among the nodes, the nodes can divided into depending type, symbiotic type and arteriovenous type.

Symbiotic type means that the nodes take advantage of the waste and by-product of others' production process through the means of exchange and then take it as the raw materials or part of the raw materials in its own production, which realizes the recycling utilization of material flow and energy.

Depending type means that there exists a leading node in the topological structure and other nodes are the auxiliary nodes derived from the leading one. The leading and auxiliary nodes form a complete set to realize the circular utilization and maximum interests. 
Arteriovenous type means that in the circular system, one kind of enterprise forms the industry chain from exploitation of raw material to production-flow-consumption-abandonment while another kind of enterprise forms the production chain by transforming the waste into renewable resources. The two kinds of enterprises share out the work and cooperate with each other like people's arteriovenous.

\section{Linear}

The linear in the topological structure of the circular economic system means the interaction among the nodes or the combined link relations and it is the key factor for the system to operate efficiently and stably.

1). In form, this link relation has two forms which are the property form and exchange form. Equity relationship is the relationship existing among the nodes. For example, Yangchun Industrial Park in Sichuan is mainly built by several enterprises subordinate to Tianyuan Group based on the principle of circular economy. Exchange relationship is the independent entities among the nodes and there only exists pure exchange relationship among them. The former is more stable than the later.

2). In content, the contents of links mainly are material and energy. The material include the waste, by-product from production process, surplus raw material, the production whose grade and standard can't meet the requirement and the instruments and equipments which are unusable or not working in the upstream enterprises. Most energy is un-renewable so how to match the energy reasonably, use it properly and improve the efficiency of energy use are the key planning contents required in the circular economy system.

\section{The Type of Topological Structure in the Circular Economic System}

According to the various nodes and geometrical shapes formed by the node links in circular economic system, the circular economic system can be divided into linear structure, circle structure, mononuclear structure, multinuclear structure and star structure, etc.

\section{Linear Structure}

Linear structure is the open-cycle economic structure of one-direction linear flow of material, energy and waste. The traditional economy pattern is the open-cycle pattern of material one-direction linear flow constituted by 'resources-production-pollutant discharge'

\section{Circle Structure}

Circular structure means that the nodes of topological happen to form a loopback to realize the closed-type feedback circular process of 'resources-production-resource regeneration-production-resource regeneration'. When designing the products, enterprise has considered the handling and circular utilization of the products after its use, which realizes the sustainable use of resources.

\section{Mononuclear Structure}

There is only one core enterprise in the circular economic system, so a series of auxiliary industries are built around it in order to take advantage of various by-products and raw materials. That kind of circular economic system is the mononuclear structure. Guigang Industrial Park in Guangxi is the classical representative. Guitang group has built a series of subsidiary corporation or branches by regarding itself as the core. It decreases the pollution through resource recycling, which realizes the economic and social interests.

\section{Multinuclear Structure}

Multinuclear structure means that circular economic system builds symbiotic internet by centering on various core enterprises. Compared with mononuclear structure, auxiliary industries have stronger independence and the topological structure is relatively stable. Karup Park in Denmark is the 
classical representative and it takes oil company's oil refinery in Norway, power station in Asness, Node drug company and Gypsum company as the core.

\section{Star Structure}

It is hard for the uniform system of resource recycling and waste disposal built in the garden to conduct reprocessing about the waste due to the cause of technology and cost so that the enterprises build connection with it. This kind of topological structure is star structure.

\section{The Formation Mode of Topological Structure}

\section{Spontaneous Formation}

Spontaneous formation means that under certain conditions, the system goes from simple to complex, disorder to order and improves constantly in level through self-adjusting and self-organization under the influence of energy utilization and mutual transformation so as to form a balanced system. The advantage of this method is reflected in: As the enterprises inside the system survive in constant competition and evolvement, they can take full advantage of material and energy surrounding them and have strong competitiveness. The disadvantage lies in: In order to pursue the maximum self-interests, enterprises perform some acts which harm the society and nature. Also the exchange instability may be increased due to its spontaneity and lack of coordination, which raises the risk of enterprise cooperation.

\section{The Planning Formation}

Planning formation means that leaded by the government, related enterprises of different industries are gathered consciously so they share the resources and form the relationship among the enterprises and systematic topological structure in government planning. The low rate of success of industrial parks formed in this way lies in that it ignores enterprises' requirement about profit and competitiveness. The basis of circular economy is resource exchange while exchange relies on market regulation instead of the direct involvement of the government.

\section{The Formation of Spontaneous Planning}

The formation of spontaneous planning means that on the basis of respecting the independent decision-making and market mechanism and through the guiding and planning of the government, the process of circular economic system which takes the increase of resource utilization rate, decrease of operation cost and the increase of economic environment earnings as the goal, is formed on the basis of resource exchange. This is a relatively ideal formation mode of the circular economic system structure. Because the formation of the circular economic operation order with high-efficient production, superior resource allocation and best restriction of economic entity behavior can't be realized from the single action of government planning or market mechanism. It must be the result of the good coupling of the two. The premise for the coupling of the two is to let the market mechanism fully play its role and then government guides, adjusts and regulates the enterprises' activities through the implementation of law, policy and system.[3]

\section{The Promotion Mechanism for Constructing the Stability of the Topological Structure of the Circular Economic System}

The stability of the topological structure of the circular economic system means the system topological structure's ability to maintain the stability when variations at different degrees occur in and outside the whole environment of the circular system. The variation in and outside the environment of the circular system is some kind of fluctuation which occurs in the operation mechanism of the whole system as the components and the environment of the outside market, economy, policy, law inside the system change. 


\section{The Analysis of the Stability of the Topological Structure}

Firstly, various nodes form the balance of material and energy transmission due to the relationship between demand and supply.

Secondly, the transmission of material and energy among the nodes can form good economic and social interests.

Thirdly, the relationship among the nodes is diversified and only the diversified relations can resist the fluctuant risk effectively.

Fourthly, the stability of topological structure is a static goal as well as a dynamic process of continuous improvements and the stability is a dynamic one.

\section{The Promotion Mechanism for Constructing the Relative Stability of Topological Structure.}

When many industrial parks are rebuilding the circular economy, the problems such as attaching importance to construction, making light of maintenance, valuing vertical coupling, neglecting lateral coupling and the unreasonable construction planning make the circular economic system very fragile. The economic and natural social interests can't be reflected and the circular economy is unworthy of its name. If this situation is to be changed, we should start from three aspects which are the construction of the appropriate operation environment for system, the perfection of the effect of various nodes and the reinforcement of the node relationship in order to generate the endogenous and outside force which stabilizes the topological structure.

The Construction of Appropriate Operation Environment for System

1). The Policy Environment

Seen from the current practice, the policy supply of circular economy is still insufficient. Government should abide by the following principles when it is implementing the policy. Firstly, it should take market mechanism as the basis and policy as the guidance. Secondly, it should bring the effect of environment protection and resource saving into the evaluation system of the government achievements. Thirdly, the misplacement of understanding and willingness between the government and the enterprise and among the government departments should be eliminated to avoid the chaos of promotion mechanism. Fourthly, apply the various policy means such as finance, revenue, price, investment, banking and government procurement and maintain the consistency comprehensively.

2). Technological Environment

There exist problems such as valuing end treatment, making light of source treatment, unsystematic technology, the obscure property right and the incomplete incentive mechanism in the circular economic technology in our country. In technological support for the strength of circular economy, on the one hand, technological systematization is required and the technology in three aspects which are business-oriented, park-oriented and city-oriented needs development in coordination. On the other hand, an efficient technological service system needs to be built and environment technology needs to be brought into the scientific tackling plan to build an expert consulting database.

3). Legal Environment

At present, the legislative status of circular economy is inconsistent with the requirement of circular economy. On the one hand, the legislation of circular economy needs to be strengthened and the behaviors of government, enterprise and residents need to be specified from aspects of constitution and environmental basic law. On the other hand, our country should further reinforce the law enforcement and law-abiding of the circular economy and legal supervision.

4). The Environment of Property Right

During the process of the practice of circular economy, the lack of property rights exist widely in four aspects including natural resources, natural environment, waste by-product and technology. The lack of property rights results in the lack of motivation of microcosmic body for the circular economy development and hampers the transfer of traditional economy into the circular economic pattern.

5). The Consumption Environment 
Production satisfies consumption and consumption counteracts production. Consumption requirement influences the production pattern and production mode of the enterprise. Therefore, as the direct beneficiary of circular economy, residents should consume green products and form the reversed mechanism which conducts circular economy for the enterprise.

The Reinforcement of Coupling Relationship

To maintain the relatively circular economic system, the coupling relationship among the enterprises in various nodes should be strengthened.

1). The Reinforcement of Intermediary Organization

During the construction of circular economy, the intermediary organization plays a great role in promotion. Countries such as Germany and Japan have all built the intermediary organization and formed the perfect recycling of renewable resources and reusing of waste. Intermediary organizations can have the effect on the following aspects: the first is the platform of waste recycling, the organization of trading platform for secondhand goods and the increase of the utilization efficiency of the products; the second is trading platform of emission rights; the third is the trading platform of circular economic technology; the fourth is the information communication platform among the enterprises and the construction of bridge for the relationship of waste and cyclic utilization of energy among the enterprises.

2). The Intensification of Industry Matching

As a close and cooperative relationship exists in aspects of resources and leftover inside the industries of the circular economy, there must be a matching mechanism on the scale of various industries. Inside the endless chain, when the surplus scale of the production in the upstream industry exceeds the absorption scale of the downstream industry and the production leftover can't be digested fully, then 'secondary leftover' is generated; when the absorption scale exceeds the leftover scale of the upstream industry and the production leftover in the circular system is not enough for the digestion of downstream industry, then 'leftover shortage' occurs.[4] No matter it's 'secondary leftover' or 'leftover shortage', it means that the circular system fails to reach a good coordination.

3). The Reinforcement of Entrance and Exit Mechanism

Build a perfect standard. Form a mechanism in which the enterprise enter the system and is knocked out of the system and the survival of the fittest in order to make the operation of circular system always maintain favorable economic and social interests.

4). Reasonable Planning and Cultivation of Circular Economic System

A reasonable topological structure should be planned and cultivated constantly according to the different industry situations of the industrial parks to make the topological structure rise to mature and stable advanced status from the low status at the beginning of the construction. Thus the links among the nodes become complicated and stable, which forms the closed-loop of material and energy.

The Reinforcement of Enterprise Action

As the consumer of energy and discharger of waster, enterprise is the basic unit of circular economy, so it is the key factor for the circular economy to realize the win-win goal of 'economic development' and 'environment development'. The principle of circular economy requires that the enterprises must build the ecological enterprises according to the idea of 'ecologicalization, zero emission and closed-loop'. The measures in the following aspects can be adopted to promote the enterprises to conduct ecological construction.

Firstly, intensify the technological input for the circular economy and introduce the professionals

Secondly, change operation principle, set up enterprise ethics and define clear responsibilities and obligation of the enterprises.

Thirdly, change business principle, carry out 'environmental operation' and embody the idea of circular economy. 


\section{Acknowledgments}

This text is the research result of the research of circular economic pattern and promotion mechanism in pour-surrounding economic development zones in Yibin(2011RY012) belonging to the project of science and technology bureau in Yibin, and the report of the evaluation model's implementation of the interactive relationship in Harbor City based on the symbiotic philosophy belonging to the project in Sichuan educational department.

\section{References}

[1] Zhu Lifang. The Research and Application of the Yacht Modeling Design Based on the Bionics of HBM. Changsha: Hunan University,2011.

[2] Wangqi. The Comparative Study at Home and Abroad of the Industrial Park Construction of Circular Economy. Xining: Qinghai University, 2012.

[3] Li Yunyan. The Coupling Effect between Market Mechanism and Government Behavior during the Development of Circular Economy. The Research of Economics and Management. 2008(4)

[4] Xiao Zhongdong, Gu Yuanxun, Sun Linyan. Theoretical Research of Symbiotic System of Manufacturing Industry. Scientific Improvement and Countermeasures. 2009(17) 